\title{
Valor da Ressonância Masnética da Coluna Vertebral Lombar com Carga na Avaliação de Estenose do Canal Vertebral $\left.{ }^{*}\right)$
}

\section{Value of the Masnetic Resonance Image of the Lumbar Spine with Load in the Evaluation of Stenosis of the Lumbar Spinal Canal}

\author{
Luiz Guilherme Hartmann ${ }^{(1)}$, Artur da Rocha Correa Fernandes ${ }^{(1)}$, Jamil Natour ${ }^{(2)}$
}

O conceito de estenose do canal vertebral é baseado no fato de que um espaço mínimo é necessário para o funcionamento normal das estruturas nervosas do canal vertebral, e que este espaço, sob certas circunstâncias, se torna estreito. Na prática clínica, um número expressivo de pacientes com sintomas significativos não possuem alterações correspondentes em exames de tomografia computadorizada (TC) e ressonância magnética (RM); para estes casos é possível que as alterações morfológicas atinjam níveis críticos e sintomatológicos durante a aplicação de carga.

A aplicação de carga axial produz efeitos dinâmicos sobre o canal vertebral, tanto em pacientes sintomáticos quanto na população normal, diminuindo a área transversal do saco dural em um grande número de pacientes com estenose do canal vertebral ${ }^{(1-7)}$.

Dentre as causas de estenose em adultos, podemos dividilas em constitucional ("congênita") e degenerativa. A estenose puramente constitucional é um fenômeno permanente, que não é influenciado significantemente pela postura ou pela carga axial do peso corporal sobre a coluna ${ }^{(8)}$. Um segmento estreito do canal vertebral pode se tornar estenótico e sintomático quando alterações degenerativas se sobrepõem a este estreitamento constitucional.

A estenose degenerativa, que se instala em um canal vertebral constitucionalmente normal, pode acometer o canal vertebral propriamente dito, sendo chamada de estenose central, ou os recessos laterais, denominada estenose lateral, isoladamente ou simultaneamente. A forma degenerativa de estenose é, em parte, um fenômeno dinâmico, influenciado pela aplicação da carga axial corporal e pela postura ${ }^{(9-11)}$. Este componente dinâmico torna importante a avaliação mor- fológica do canal vertebral e de suas modificações durante a aplicação de carga axial e de manobras dinâmicas.

Os sintomas da estenose são freqüentemente desencadeados na extensão do quadril e da coluna, posição ortostática ou em atividades de caminhada. Caracteristicamente, estes pacientes têm menos sintomas quando estão em posição de flexão da coluna ou deitados em posição supina com o quadril em flexão. Estas alterações dos sintomas relacionados à posição têm levado diversos autores a pensar que a realização de exames na posição de maior sintomatologia pode dar mais informações diagnósticas do que nos exames com o psoas relaxado. A RM e a TC são, em sua grande maioria, realizadas com o paciente na posição supina, muitas vezes com o quadril e o joelho parcialmente fletidos, que aumentam o conforto do paciente, mas que relaxam o psoas e que diminuem bastante as tensões e a carga sobre a coluna.

Quando a mielografia era o exame de escolha para a investigação de estenose do canal, a avaliação dinâmica era mais comum, realizando-se manobras dinâmicas na posição ortostática. Com o advento da TC e da RM, por permitirem estudo morfológico direto do disco, das partes ósteo-ligamentares e das estruturas nervosas de forma menos invasiva, diminuíram as solicitações de mielografia, perdendo-se a possibilidade do exame dinâmico da coluna vertebral.

Willen et al., em diversos trabalhos, têm estudado os efeitos dinâmicos da aplicação de carga axial sobre o canal vertebral, tanto em pacientes sintomáticos quanto na população normal $^{(1-3)}$, por meio de um instrumento constituído por um colete no ombro fixado em um apoio nos pés por meio de tiras, regulando-as para que seja aplicada uma força de $50 \%$ do peso corporal, quando o paciente estender os joelhos e os quadris (Figura 1).

* Trabalho realizado no Departamento de Diagnóstico por Imagem da Universidade Federal de São Paulo - Escola Paulista de Medicina (DDI-Unifesp-EPM), São Paulo, SP, Brasil.

1. DDI-Unifesp-EPM.

2. Disciplina de Reumatologia da Unifesp-EPM.

Endereço para correspondência: Dr. Artur R. C. Fernandes. Departamento de Diagnóstico por Imagem da Universidade Federal de São Paulo - Escola Paulista de Medicina (DDI-Unifesp-EPM). Rua Botucatu, 740, CEP 04023-900. São Paulo, SP, Brasil. 


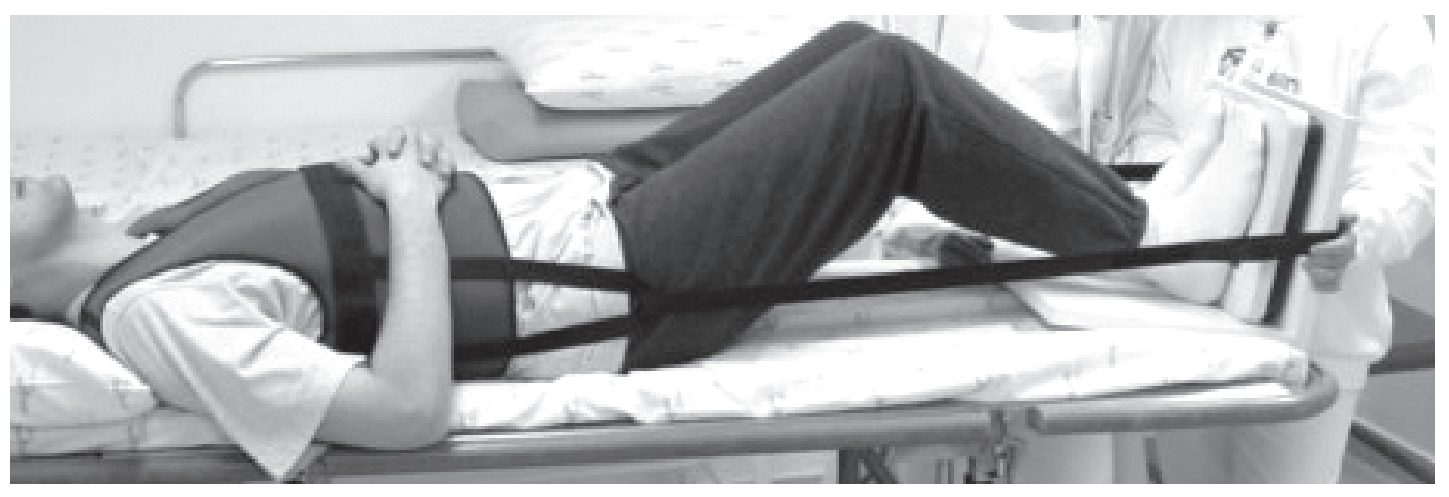

FiguRA 1 - Posicionamento do paciente durante a realização do exame de RM da coluna lombar com aplicação de carga axial. 0 paciente veste um colete com tiras amarradas a um apoio nos pés, reguladas previamente para que seja exercida uma força de compressão equivalente a 50\% de seu peso corporal, com extensão dos quadris e joelhos

Estes estudos têm mostrado que a aplicação de carga axial diminui a área transversal do saco dural em um grande número de pacientes com estenose do canal vertebral ${ }^{(6-12)}$. A razão desta acentuação da estenose é o espessamento do ligamento amarelo, a acentuação de abaulamentos ou protrusões discais e o espessamento da gordura epidural posterior, que resultam em deformação do saco dural e redução de sua área transversal. Outras modificações também podem ocorrer, como acentuação de listeses, redução dos recessos laterais e acentuação ou aparecimento de cistos sinoviais interapofisários projetando-se no canal vertebral ${ }^{(1)}$ (Figura 2).

Willen e Danielson ${ }^{(4)}$ demonstraram que a aplicação de carga axial forneceu informações adicionais relevantes no exame de imagem em $29 \%$ dos pacientes com sintomas de estenose com exames de rotina inconclusivos (Figura 3).

Um estudo mais recente de Hiwatashi et al. ${ }^{(12)}$ mostrou que estas informações adicionais fornecidas no exame com carga tiveram influência sobre a conduta clínica e cirúrgica de pacientes com estenose do canal vertebral. Nos 20 pacientes com estudo com carga axial positiva, em cinco casos ( $25 \%$ do total) três neurocirurgiões mudaram a conduta de conservadora para cirúrgica, baseados nos achados adicionais deste estudo; em outros cinco casos $(25 \%)$ houve mudanças de conduta sugeridas por um ou dois neurocirurgiões, porém sem consenso entre os três.
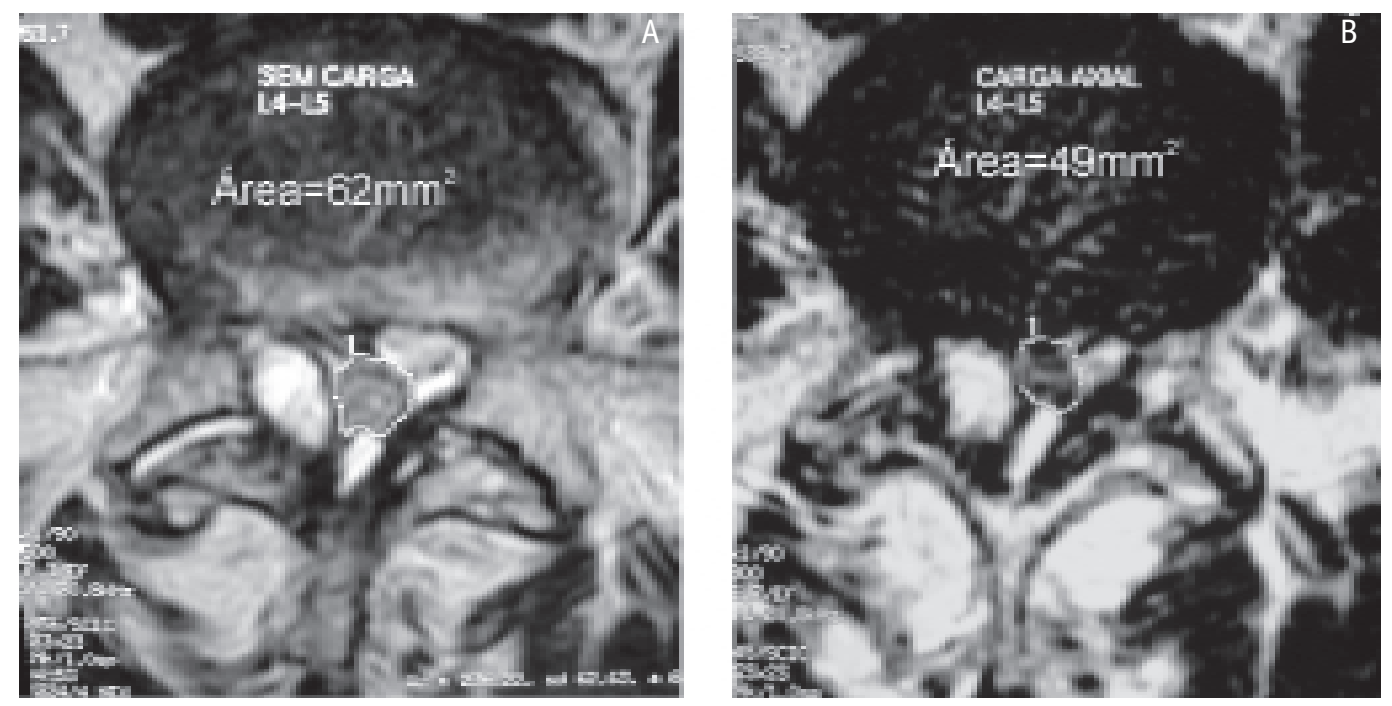

FiguRA 2 - Exemplo de alterações significativas observadas durante a aplicação de carga axial. Paciente com cisto sinovial em articulação zigoapofisária, que se projeta para o canal vertebral (A). Quando é aplicada a carga axial, o volume do cisto aumenta, o que determina maior estenose do canal, com redução significativa da área do saco dural de $62 \mathrm{~mm}^{2}$ (repouso) para $49 \mathrm{~mm}^{2}$ (carga) 

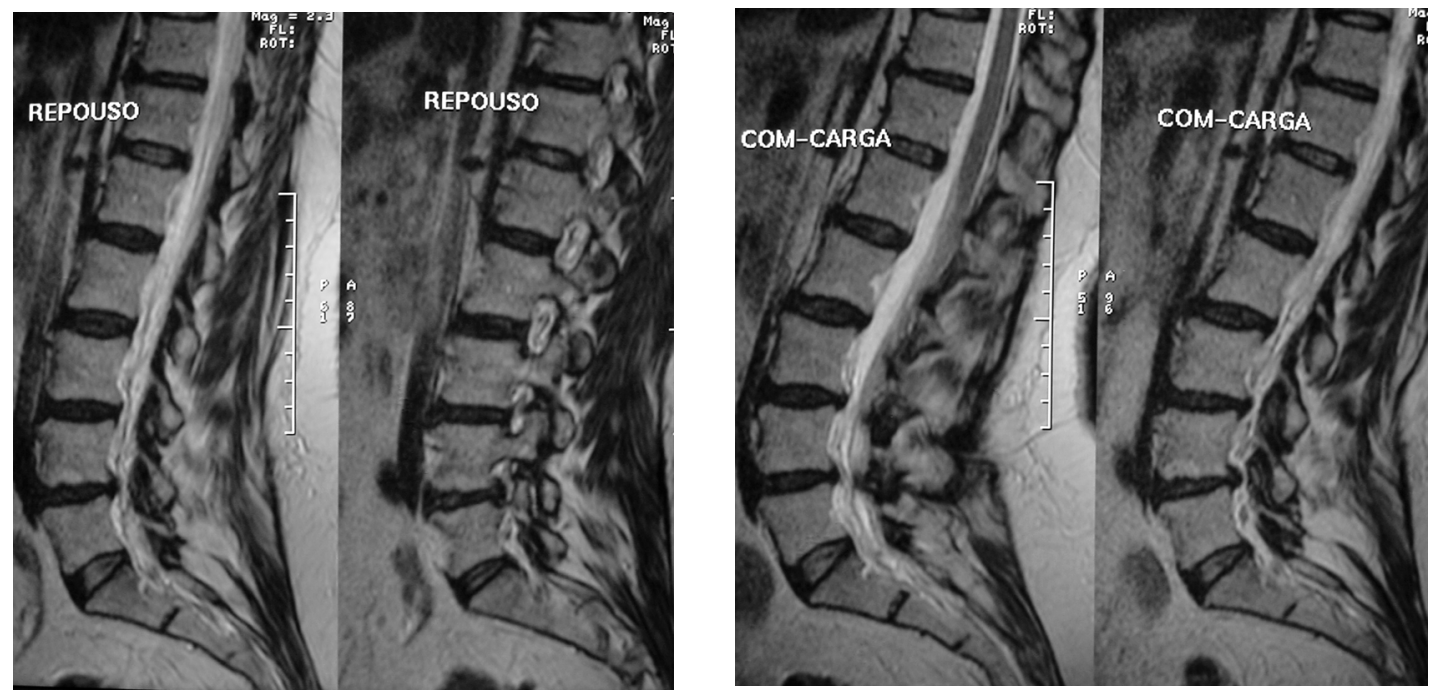

FiguRA 3 - Exemplo de alterações significativas observadas durante a aplicação de carga axial. Paciente com protrusões discais discretas e sem desvios significativos da coluna na posição em repouso. Durante a aplicação de carga, observa-se aparecimento de retrolistese L2-L3, bem como acentuação das protrusões discais

Em resumo, a RM da coluna lombossacral com carga axial é um método não invasivo que permite a avaliação dinâmica da coluna lombar, devendo ser lembrada como um estudo adicional quando a RM convencional não é capaz de justificar o quadro clínico e os sintomas de pacientes com sinais de estenose do canal vertebral, podendo

\section{REFERÊNCIAS}

1. Willén J, Danielson B, Gaulitz A, Nicklason T, Shönstrom N, Hansson T: Dynamic effects on the lumbar spinal canal: axially loaded CT-myelography and MRI in patients with sciatica and/or neurologic claudication. Spine 22: 2968-76, 1997.

2. Danielson B, Willén J, Gaulitz A, Niklason T, Hansson TH: Axial loading of the spine during $\mathrm{CT}$ and $\mathrm{MR}$ in patients with suspected lumbar spinal stenosis. Acta Radiol 39: 604-11, 1998.

3. Danielson B, Willén J: Axially loaded magnetic resonance image of the lumbar spine in asymptomatic individuals. Spine 26: 2601-6, 2001.

4. Willén J, Danielson B: The diagnostic effect from axial loading of the lumbar spine during computed tomography and magnetic resonance imaging in patients with degenerative disorders. Spine 26: 2596-600, 2001

5. Kimura S, Steinbach GC, Watenpaugh DE, Hargens AR: Lumbar spine disc height and curvature responses to an axial load generated by a compression device compatible with magnetic resonance imaging. Spine 26: 2596-2600, 2001.

6. Schmid MR, Stucki G, Duewell S, Wildermuth S, Romanowski B, Hodler J; Changes in cross-sectional measurements of the spinal canal ser uma alternativa à mielografia dinâmica. A aplicação da carga axial pode fornecer informações adicionais relevantes que servem tanto para o diagnóstico quanto para a tomada de conduta desses pacientes. Devemos salientar, entretanto, que mais estudos devem ser realizados para validar a RM com carga axial como exame indicado nesses casos.

and intervertebral foramina as a function of body position: in vivo studies on an open-configuration MR system. AJR Am J Roentgenol 172: 1095-102, 1999.

7. Weishaupt D, Schmid MR, Zanetti M, et al: Positional MR imaging of the lumbar spine: does it demonstrate nerve root compromise not visible at conventional MR imaging? Radiology 215: 247-53, 2000.

8. Schönström N, Willén J: Imaging lumbar spinal stenosis. Radiol Clin N Am 39: 31-53, 2001.

9. Penning L, Wilminck JT: Biomechanics of the lumbosacral dural sac: a study of flexion-extension myelography. Spine 6: 398-408, 1981.

10. Sortland O, Magnaes B, Hauge T: Functional myeloraphy with metrizamide in the diagnosis of lumbar spinal stenosis. Acta Radiol Suppl 355: 42-54, 1977.

11. Wilson CB, Ehni G, Grollmus J: Neurogenic intermittent claudication. Clin Neurosurg 18: 62-85, 1971.

12. Hiawatashi A, Danielson B, Moritani T, et al: Axial loading during MR Imaging can influence treatment decision for symptomatic spinal stenosis. AJNR Am J Neuroradiol 25: 170-4, 2004. 